\title{
Study Reference
}

National Cancer Institute

\section{Source}

National Cancer Institute. Study Reference. NCI Thesaurus. Code C93452.

A citation to a publication related to the protocol's background. 